\title{
Neuro-genetic plasticity of Caenorhabditis elegans behavioral thermal tolerance
}

\author{
Gregory W. Stegeman ${ }^{1}$, Denise Medina ${ }^{1,2}$, Asher D. Cutter ${ }^{1 *}$ and William S. Ryu ${ }^{2,3^{*}}$ (D)
}

\begin{abstract}
Background: Animal responses to thermal stimuli involve intricate contributions of genetics, neurobiology and physiology, with temperature variation providing a pervasive environmental factor for natural selection. Thermal behavior thus exemplifies a dynamic trait that requires non-trivial phenotypic summaries to appropriately capture the trait in response to a changing environment. To characterize the deterministic and plastic components of thermal responses, we developed a novel micro-droplet assay of nematode behavior that permits information-dense summaries of dynamic behavioral phenotypes as reaction norms in response to increasing temperature (thermal tolerance curves, TTC).

Results: We found that C. elegans TTCS shift predictably with rearing conditions and developmental stage, with significant differences between distinct wildtype genetic backgrounds. Moreover, after screening TTCs for 58 C. elegans genetic mutant strains, we determined that genes affecting thermosensation, including $\mathrm{cmk}-1$ and tax-4, potentially play important roles in the behavioral control of locomotion at high temperature, implicating neural decision-making in TTC shape rather than just generalized physiological limits. However, expression of the transient receptor potential ion channel TRPA- 1 in the nervous system is not sufficient to rescue rearing-dependent plasticity in TTCs conferred by normal expression of this gene, indicating instead a role for intestinal signaling involving TRPA- 1 in the adaptive plasticity of thermal performance.
\end{abstract}

Conclusions: These results implicate nervous system and non-nervous system contributions to behavior, in addition to basic cellular physiology, as key mediators of evolutionary responses to selection from temperature variation in nature.

Keywords: C. elegans behavior, Thermal ecology, Computational ethology

\section{Background}

Behaviors are the primary way that animals interact with their environments and, in so doing, connect genetics and physiology with ecology. Natural selection favours those alleles of genes that allow animals to sense stimuli and react in ways that maximize fitness [1,2]. While many aspects of behavior are heritable, it remains an ongoing challenge to discover mechanisms of when and where genes will influence specific aspects of behavior [3]. Behaviors as phenotypes are inherently complex

\footnotetext{
*Correspondence: asher.cutter@utoronto.ca; willryu@gmail.com ${ }^{1}$ Department of Ecology and Evolutionary Biology, University of Toronto, Toronto, Canada

${ }^{2}$ Department of Physics, University of Toronto, Toronto, Canada Full list of author information is available at the end of the article
}

because of the combination of environmental and genetic factors that influence them, ranging from internal and external conditions of an individual, to widespread functional pleiotropy and gene by environment interactions, to previous experience of individuals and their social interactions $[4,5]$. Because many behavioral responses are reactions to stimuli, it is natural to describe a behavioral trait as a functional response, the norm of reaction to a range of environmental inputs $[6,7]$. Temperature provides a ubiquitous environmental input that is particularly crucial to organismal fitness and to behavior, commonly characterized phenotypically as thermal performance curves [8-10]. Behavior is the primary strategy of ectothermic animals to regulate their body temperatures, with sensing, orienting and navigating the thermal

C The Author(s) 2019. This article is distributed under the terms of the Creative Commons Attribution 4.0 International License (http://creativecommons.org/licenses/by/4.0/), which permits unrestricted use, distribution, and reproduction in any medium, provided you give appropriate credit to the original author(s) and the source, provide a link to the Creative Commons license, and indicate if changes were made. The Creative Commons Public Domain Dedication waiver (http://creativecommons.org/ publicdomain/zero/1.0/) applies to the data made available in this article, unless otherwise stated. 
landscape being critical for fitness [11, 12]. Invention of quantitative behavioral assays has helped geneticists unravel the roles of genes in behavior by controlling as many variables as possible and by quantifying behavioral traits as accurately as possible [13]. In order to quantify the dynamic temperature-dependent behavior of Caenorhabditis nematode and to decipher its neuro-genetic control, we developed a novel assay to quantify locomotory behavior through a range of ecologically relevant temperature.

As small-bodied ectotherms, C. elegans worms regulate their body temperature through movement, making the sensing and orienting to thermal stimuli crucial for survival and reproduction in nature. C. elegans navigates its thermal landscape predominately through two behaviors: thermotaxis [14] and thermal avoidance [15, 16]. Although much is known about the molecular and cellular mechanisms of thermosensation and thermosensory neural circuits in C. elegans [17], much less is known about the general adaptive mechanisms that $C$. elegans uses as it explores its entire ecological temperature range. Powerful approaches to study temperature-dependent behaviors in C. elegans include "classic" assays in Petri dishes as well as automated and microfluidic measurement [18-20], but none of these assays have characterized C. elegans thermal performance curves. Our goals led us to develop a new assay for C. elegans temperature-dependent behavior to quantify thermal tolerance from video recordings, where we capture the behavioral responses by a given genotype across a range of temperatures as a 'norm of reaction.

By framing the problem of understanding behavior as a set of thermal reaction norms in the genetically-tractable C. elegans system, we aimed to answer key questions about deterministic and plastic contributors to behavior. How do genes and sensory inputs modulate behavioral thermal performance? Do neural decisions or fundamental physiological limitations drive behavioral performance at high temperatures? How sensitive is latelife thermal performance to early-life experience and can genes modulate that sensitivity? In addressing these outstanding questions, we aim to decipher the links between thermal ecology and the neural and genetic controls over complex behavioral responses to stimuli.

After constructing and implementing a micro-droplet assay device for locomotory thermal performance in C. elegans, we demonstrate its efficacy for quantifying behavioral differences among natural isolates and a panel of gene mutants. By quantifying the reaction norm of the behavioral response to precisely controlled changes in temperature (thermal tolerance curve, TTC), these experiments provide evidence for a neural "decision" by animals to stop locomotion as temperature increases.
Animals with genetic defects in thermosensation shift the decision-making process to continue swimming at higher than normal temperatures or to cease moving at lower than normal temperatures. Therefore, the declining locomotory behavior with increases in temperature does not solely reflect high-temperature physiological limits, as usually presumed for other organisms [10]. Plasticity in animal TTC profiles derives from differences in age and rearing conditions. We also determined that nonneural regulation of TTCs is important, particularly for plasticity of TTC profiles in response to larval rearing conditions, with intestinal expression of the trpa-1 ion channel implicated in this process.

\section{Results}

\section{Caenorhabditis elegans swimming behavior as a thermal reaction norm}

We developed a micro-droplet assay of nematode worm swimming behavior that permits relatively high-throughput and information-dense quantification of individual locomotion in response to precise temperature manipulation. We then characterized animal movement as norms of reaction, finding that nematode behavior follows the pattern of a classic thermal performance curve in the high temperature range $[12,21]$ with locomotion most rapid at benign temperatures and steeply dropping off in performance as temperature increases until reaching paralysis (Fig. 1a). By contrast, constant benign temperatures yield much smaller, albeit significant, changes of motility over time through the course of a $21 \mathrm{~min}$. assay (Additional file 1: Fig. S3B) (repeated measures ANOVA $\left.\mathrm{F}_{19,7}=4.21, p<0.0001\right)$. Worms are slightly more active when they experience a constant $25{ }^{\circ} \mathrm{C}$ compared to $21{ }^{\circ} \mathrm{C}$ or $23{ }^{\circ} \mathrm{C}$ (repeated measures ANOVA $\mathrm{F}_{2,92}=7.96$, $P=0.0006$; also apparent in the dynamic TTC profile, Additional file 1: Fig. S3B).

Temperature-induced paralysis is reversible and the dynamics of recovery happen at multiple time scales. We tested reversibility by submitting worms to brief $80 \mathrm{~s}$, high temperature exposures $\left(35-43^{\circ} \mathrm{C}\right)$. During exposure to temperatures $>39^{\circ} \mathrm{C}$, all worms quickly ceased movement (Fig. 1b), but when returned to a benign temperature $\left(23^{\circ} \mathrm{C}\right.$, rearing temperature), worms recovered in a manner inversely related to the exposure temperature. Worms exposed to $35.6{ }^{\circ} \mathrm{C}$ rapidly recovered to control activity but those exposed to $43.1{ }^{\circ} \mathrm{C}$ did not recover at all. For intervening exposure temperatures, worms surprisingly showed non-monotonic recovery with an early phase of partial or weak recovery and then a second phase of recovery later in the assay (Fig. 1b). However when we extended the room temperature observation to 77 min post-exposure, we observed that animals exposed to $41.0{ }^{\circ} \mathrm{C}$ and $42.0{ }^{\circ} \mathrm{C}$, which recovered within a few 

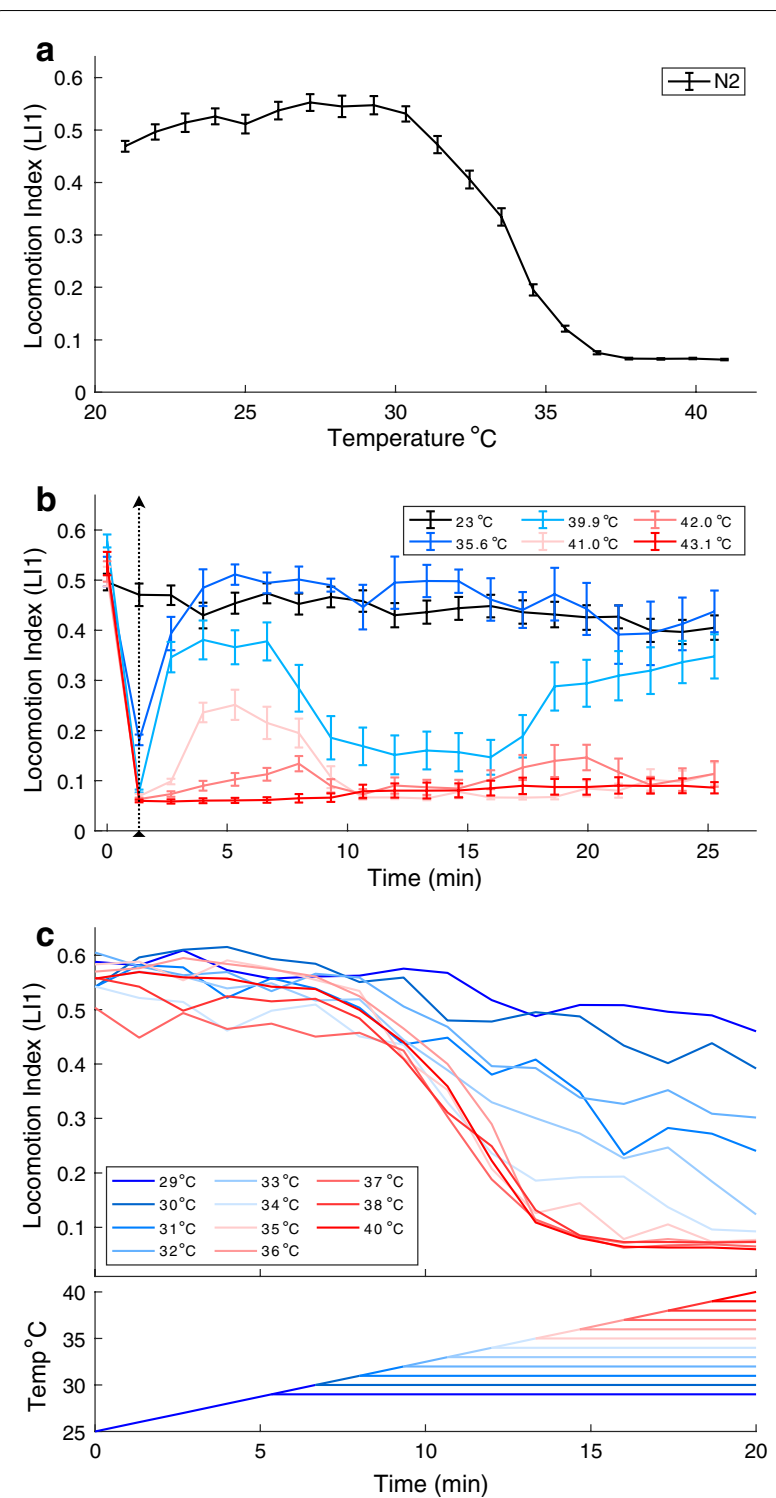

Fig. 1 Thermal tolerance curve for wildtype C. elegans. a C. elegans thermal tolerance curve. Locomotion index (LII) with temperature increments from 21 to $41^{\circ} \mathrm{C}$. Wildtype N2 adult hermaphrodite animals were reared at $23^{\circ} \mathrm{C}$. 54 worms and $\geq 35$ animals retained in calculations at each temperature step. $\mathbf{b}$ C. elegans locomotion index profiles in response to acute high temperature exposure from baseline $23^{\circ} \mathrm{C}$ (black arrow dotted line, 80 s exposure). Error bars indicate \pm SEM. 54 individuals tested per series; $\geq 34$ retained in calculations at each step. $\mathbf{c}$ Locomotion index over time at constant hold temperature after incrementing up from an initial $25^{\circ} \mathrm{C}$ (upper half of panel). Hold temperature shown on the bottom half of the panel, with hold temperature of $40^{\circ} \mathrm{C}$ comparable to the standard TTC assay; 18 worms tested per series, $\geq 9$ worms included in LI calculations at each step

minutes, ultimately ceased locomotion after about an hour (Additional file 1: Fig. S3).
Thermal paralysis can be induced over time at temperatures that are below the thermal paralysis threshold. To test sub-threshold temperatures, we increased temperature incrementally from $25{ }^{\circ} \mathrm{C}$ as before, but then held it constant for the remainder of the assay at various elevated temperatures. We found that worm locomotion declines toward immobility for any 'hold temperature' above $35^{\circ} \mathrm{C}$ (Fig. 1c). Even though worms swim at the start of the $35.6{ }^{\circ} \mathrm{C}$ step, their eventual paralysis does not require that they experience higher temperatures. In fact, worms exposed to hold temperatures of $\sim 33^{\circ} \mathrm{C}$ also come close to paralysis within the $21 \mathrm{~min}$ assay, whereas hold temperatures less than $\sim 30{ }^{\circ} \mathrm{C}$ exert at most a minimal effect on locomotion (Fig. 1c). These experiments implicate a cumulative effect of temperature exposure above a critical threshold of $\sim 30{ }^{\circ} \mathrm{C}$, and a delay in the ultimate outcome of exposure to temperatures higher than $\sim 33{ }^{\circ} \mathrm{C}$. In agreement with our work, previous research on heat shock showed that a 20 min exposure to $40{ }^{\circ} \mathrm{C}$ led to $\mathrm{C}$. elegans quiescence, a sleep-like state, lasting several hours [22] and $<25 \%$ of worms survived a 15 min exposure to $39{ }^{\circ} \mathrm{C}$ [23]. These results show that the TTC is a complicated convolution of thermal thresholds and cumulative effects, and so the precise shape of the TTC depends on the time spent at each target temperature.

\section{Plasticity of $C$. elegans behavioral thermal tolerance curves: rearing conditions and life stage}

In order to place the micro-droplet thermal performance assay in a broader ecological context, it is important to consider other life stages and rearing conditions. Given how strongly rearing temperature affects growth rates and other aspects of C. elegans biology, as well as the role of thermal acclimation and memory in thermotaxis and isothermal tracking behaviors [24-27], we expected rearing temperature to affect thermal tolerance curves of locomotory behavior. We quantified this sensitivity by comparing $\mathrm{N} 2$ worms reared at $15^{\circ} \mathrm{C}, 20^{\circ} \mathrm{C}, 23^{\circ} \mathrm{C}$ and $25^{\circ} \mathrm{C}$ in terms of their TTCs as the animals experienced temperature increments from 21 to $40{ }^{\circ} \mathrm{C}$ (Fig. 2a). We found that cooler rearing temperatures led to more rapid declines in swimming behavior as we raised the temperature (Fig. 2a). Opposing this trend, however, C. elegans reared at $25{ }^{\circ} \mathrm{C}$ exhibit reduced locomotion compared to worms reared at $23{ }^{\circ} \mathrm{C}$. We hypothesize that animals reared at $25{ }^{\circ} \mathrm{C}$ may have a developmental fate difference that alters the TTC because this higher temperature is known to compromise C. elegans fecundity [28, 29].

We also contrasted adult TTCs with those of larvae and found that animals in the two final larval stages (L3 and L4) slow their locomotion more readily than do adults in response to increasing temperatures, even at 


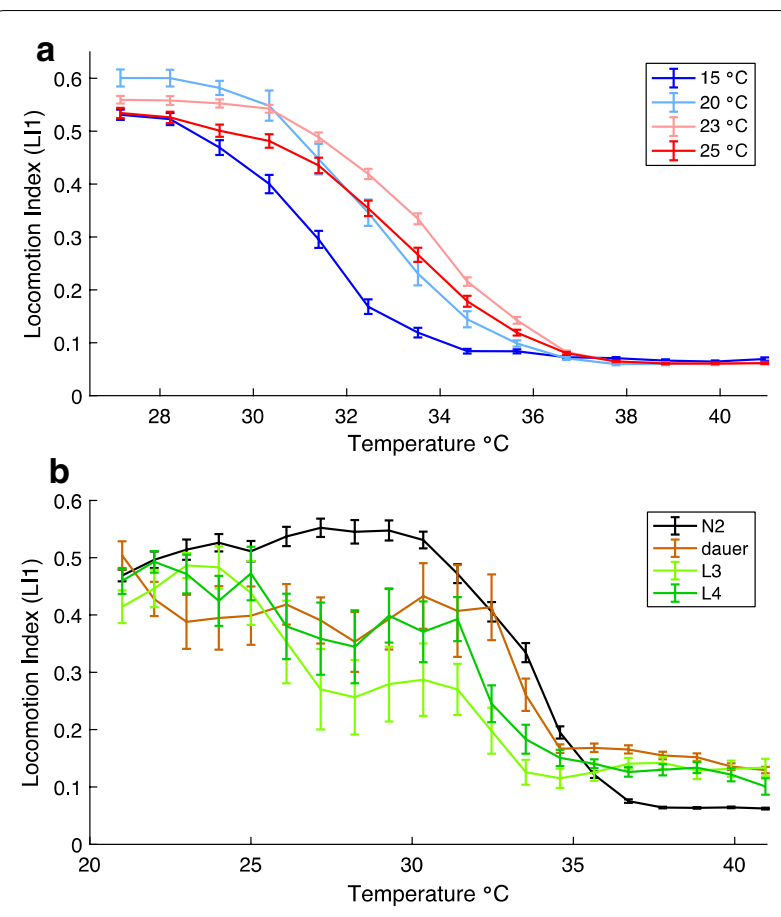

Fig. 2 Effect of rearing temperature and developmental stage on C. elegans TTC. a Rearing conditions at both high and low thermal extremes shift wildtype N2 C. elegans thermal performance curves relative to rearing at intermediate thermal conditions. 36-114 animals tested per thermal rearing condition, $\geq 16$ individuals included in calculations at each assay step. b TTCs for adult and larval developmental stages. 9-27 worms tested per treatment, $\geq 5$ worms included in calculations at each assay step. Error bars indicate \pm SEM

relatively benign temperature points $\left(\sim 26^{\circ} \mathrm{C} ; \mathrm{F}_{2,62}=8.49\right.$, $P=0.0006$, Dunnett's post hoc test L3 $P=0.0021$, L4 $P=0.0072$; Fig. 2b). The L3 animals tend to move on average more slowly than L4 animals and essentially stop movement above $33{ }^{\circ} \mathrm{C}$, whereas L4 worms continue some movement up to $35.6{ }^{\circ} \mathrm{C}$ (Fig. 2b). By contrast, dauer larvae tend to move at a slow and consistent pace at benign temperatures up to $32{ }^{\circ} \mathrm{C}$ when they start to slow at a rate similar to adult animals (Fig. 2b). Dauer larvae are a long-lived stress resistant alternate larval pathway for many nematode species including $C$. elegans, capable of surviving higher temperatures than normal larvae [30,31]. These experiments point to different developmental stages as having distinct TTC shapes, perhaps reflecting different optimal strategies for actively developing animals, distinct neural processing circuitry, or different capability of handling temperature stress.

\section{Genetic perturbation of C. elegans behavioral thermal tolerance curves}

Many C. elegans genes affect behaviors, so we screened 58 gene mutant strains (Table 1) to see how these genes might change behavioral thermal performance compared to two "wildtype" control strains in the micro-droplet assay, with temperature incrementing from 27 to $40{ }^{\circ} \mathrm{C}$. In particular, we aimed to analyze mutants with known phenotypic disruptions of thermotaxis, thermosensation, mechanosensation, chemosensation, locomotion, and neural function (Fig. 3 and Additional file 1: S4). The Hawaiian wildtype strain (CB4856) appears to slow movement more sharply above $33{ }^{\circ} \mathrm{C}$ than the N2 (Bristol) strain, but nevertheless continues locomotion to the same high temperatures as $\mathrm{N} 2$, with comparable locomotion index scores above $36{ }^{\circ} \mathrm{C}$ (Fig. 3b). The TTC for the $n p r-1$ mutant performs similar to CB4856 (Fig. 3b), which has a functionally similar allele of $n p r-1$ [32]. Most mutant strains exhibited lower locomotion indices at high temperatures compared to the wildtype N2 $\left(\mathrm{F}_{59,1550}=10.79, P<0.0001,47\right.$ mutant strains lower and only cmk-1 higher than wildtype with Dunnett's post hoc tests $P<0.05$ at $35.6{ }^{\circ} \mathrm{C}$; Fig. 3), including the other wildtype strain (N2 LI1 $=0.56$ vs. CB4856 LI $1=0.48$ at $27.2^{\circ} \mathrm{C}$ ). Indeed, about half of the mutants show very low activity even under benign conditions, consistent with them having basic roles in locomotion. In general, it is difficult to dissect the mechanistic implications of strains with very poor locomotion because we cannot easily distinguish general locomotory defects from those caused by perturbation specific to thermal paralysis. However, we can use the condition of thermal paralysis to compare curves, even for those with initial low activity. While most curves share the same shape as the wild-type TTC, some curves reach paralysis at a much higher temperature (dgk-1, goa-1, mec-6, tax-4, unc-46; $\mathrm{F}_{59,1540}=9.64$, $P<0.0001$, Dunnett's post hoc tests $P<0.02$ at $37.8{ }^{\circ} \mathrm{C}$ ) (Fig. 3b) or lower temperature (mec-3, odr-1, odr-3, pkc1, daf-21, hsf-1, all Dunnett's post hoc test $P<0.0001$ at $35.6{ }^{\circ} \mathrm{C}$ ) (Fig. $3 \mathrm{c}$ ), suggesting non-trivial perturbations affecting the TTC. And finally, we had hypothesized that a number of mutants would have strongly altered TTCs, but their TTCs were relatively unchanged compared to the wildtype (e.g. hsf-16.48) (Fig. 3b).

\section{Neuronal gene disruptions can enhance or reduce TTC responses}

The eight thermosensory mutants provide a class of mutations of special interest (Fig. 3, Additional file 1: Fig. S4B). Might a neural "decision" act to stop locomotion as temperature increases? We hypothesized that if neural control drives the slowing of locomotion at higher temperatures, then thermosensory mutant strains should exhibit distinct TTCs compared to wildtype and implicate sensation or perception of temperature in the response rather than some fundamental physiological limit. 
Table 1 Mutant strains quantified for differences in temperature-dependent locomotory behavior

\begin{tabular}{|c|c|c|c|}
\hline Strain & Mutant gene(s) & Allele & Mutation type ${ }^{a}$ \\
\hline N2 & wildtype Bristol & & \\
\hline CB4856 & wildtype Hawaii & & \\
\hline TJ1052 & age-1 & $h \times 546$ & Substitution \\
\hline CB6055 & bus-8 & e2698 & Substitution \\
\hline CB1112 & cat-2 & e1112 & Substitution \\
\hline TN101 & cha-1 & cn101 & From EMS \\
\hline CB1124 & che-3 & e1124 & Substitution \\
\hline PY1589 & cmk-1 & oy21 & Substitution \\
\hline PR673 & daf-21 & p673 & From EMS \\
\hline RM2702 & dat-1 & ok157 & From UV/TMP \\
\hline JT748 & dgk-1 & sa748 & From EMS \\
\hline LX636 & dop-1 & vs101 & Deletion \\
\hline LX703 & dop-3 & vs106 & $\begin{array}{l}\text { Deletion in promotor } \\
\text { and 1st transmem- } \\
\text { brane domain }\end{array}$ \\
\hline BZ33 & dys-1 & eg33 & From EMS \\
\hline DA572 & eat-4 & ad572 & From $X$-ray \\
\hline MT1212 & egl-19 & n582 & Substitution \\
\hline VC461 & egl-3 & gk238 & Deletion \\
\hline IK597 & $\begin{array}{l}\text { gсу-23; gсy-8; } \\
\text { gсу- } 18\end{array}$ & nj37, oy44, nj38 & All deletions \\
\hline IK800 & gсу-8 & oy44 & Deletion \\
\hline KP4 & $g \mid r-1$ & n2461 & Substitution \\
\hline RB1808 & $g \mid r-2$ & ok2342 & Deletion \\
\hline JT734 & goa-1 & sa734 & Substitution \\
\hline PS3551 & hsf-1 & sy441 & Substitution \\
\hline RB791 & hsp-16.48 & ok577 & Deletion \\
\hline MT382 & $\operatorname{lin}-11$ & n382 & From EMS \\
\hline CB1515 & mec-10 & e1515 & Substitution \\
\hline CB1338 & mec-3(e1338) & e1338 & Insertion-frameshift \\
\hline VC2396 & mec-3(gk1126) & gk1126 & Insertion/deletion \\
\hline CB1339 & mec-4 & e1339 & Substitution \\
\hline CB1472 & mec-6 & e1342 & Substitution \\
\hline DA609 & npr-1 & ad609 & Substitution \\
\hline CX2065 & odr-1 & n1936 & From EMS \\
\hline CX2205 & odr-3 & $\mathrm{n} 2150$ & From EMS \\
\hline МT3631 & osm-3 & n1545 or n1540 & From EMS \\
\hline PR811 & osm-6 & p811 & Substitution \\
\hline IK105 & pkc-1 & nj1 & Substitution \\
\hline PR671 & $\operatorname{tax}-2$ & p671 & Substitution \\
\hline FK129 & $\operatorname{tax}-4(\mathrm{ks} 11)$ & ks 11 & Substitution \\
\hline PR678 & $\operatorname{tax}-4(p 678)$ & p678 & Substitution \\
\hline PR675 & $\operatorname{tax}-6$ & p675 & Substitution \\
\hline GR1321 & tph-1; cam-1 & mg280 \& vs166 & Deletion \\
\hline VC160 & $\operatorname{trp}-1$ & ok323 & Deletion \\
\hline RB1052 & trpa-1 & ok999 & Uncurated \\
\hline PR767 & $\mathrm{ttx}-1$ & p767 & Substitution \\
\hline FK134 & $\mathrm{ttx}-3$ & ks5 & Substitution \\
\hline IK575 & $\mathrm{ttx}-7$ & nj40 & Substitution \\
\hline
\end{tabular}

Table 1 (continued)

\begin{tabular}{llll}
\hline Strain & Mutant gene(s) & Allele & Mutation type $^{\mathbf{a}}$ \\
\hline TN110 & twk-18 & cn110 & Substitution \\
MT1684 & unc-105(n490n785) & n490n785 & Double mutation \\
MT1098 & unc-105(n506) & n506 & Uncurated \\
VC854 & unc-2 & gk366 & Deletion \\
BC18 & unc-22 & s13 & From EMS \\
CB403 & unc-29 & e403 & Substitution \\
CB251 & unc-36 & e251 & Substitution \\
ZZ20 & unc-38 & x20 & Uncurated \\
CB177 & unc-46 & e177 & Substitution \\
CB382 & unc-49 & e382 & Substitution \\
BC347 & unc-54 & s74 & Substitution \\
CB1068 & unc-79 & e1068 & From EMS \\
MT1085 & unc-8 & $n 491$ & Substitution \\
CB1069 & unc-80 & e1069 & From EMS \\
MT200 & unc-93 & $\mathrm{n} 200$ & From EMS \\
\hline
\end{tabular}

a Mutation information from wormbase.org

Indeed, we found that two thermosensory mutant genotypes outperform the wildtype over part of the thermal tolerance curve [cmk-1(oy21) at $34.6{ }^{\circ} \mathrm{C} \quad \mathrm{F}_{59,1563}=14.39, \quad P<0.0001$, Dunnett's post hoc test $P<0.0001 ; \operatorname{tax}-4(\mathrm{p} 678)$ at $37.8{ }^{\circ} \mathrm{C}$ as above $\mathrm{F}_{59,1540}=9.64, \quad P<0.0001$, Dunnett's post hoc tests $P<0.0001$; Fig. 3b]. However, cmk-1(oy21) and tax4(p678) differ in the trend of how they manifest higher locomotion than wildtype at high temperatures. The cmk-1(oy21) TTC is shifted toward hot temperatures whereas the $\operatorname{tax}-4$ (p678) TTC shows a nearly linear decay from benign temperatures that eventually yields higher locomotion than $\mathrm{N} 2$ from 36 to $39{ }^{\circ} \mathrm{C}$ (Fig. 3b). Interestingly, an alternative allele of $\operatorname{tax}-4(\mathrm{ks} 11)$ that is a missense rather than a nonsense mutation [33] does not show elevated movement at high temperatures like the p678 allele (Dunnett's post hoc test $P=0.12$ for tax$4(\mathrm{ks} 11)$ at $37.8^{\circ} \mathrm{C}$ ) (Additional file 1: Fig. S4B). Both of these loci disrupt development of AFD, the main thermosensory neuron in C. elegans [34]. The fact that these AFD-expressed thermosensory genes (cmk-1, tax-4) both disrupt TTCs, either positively or negatively, implicates neuronal thermal sensory measurement as important in the thermal locomotory performance and indicates that reduced high-temperature locomotion is not simply a result of general physiological limits.

Given the neural connection to TTCs, we explored whether mutations that alter neural circuitry or sensory structures that impact mechanosensation and chemosensation might also disrupt TTCs. Of these 22 mutants, five became paralyzed at lower temperatures than N2 (mec-3, odr-1, odr-3, pkc-1, daf-21; all Dunnett's post hoc 
test $P<0.0001$ at $35.6{ }^{\circ} \mathrm{C}$ ) (Fig. 3c). The other 18 strains exhibited deficits in thermal performance at moderately high temperatures $\left(32-36^{\circ} \mathrm{C}\right)$, but most were comparable in TTC profile to the CB4856 Hawaiian wildtype strain (7/11 mechanosensory and 10/11 chemosensory mutants lower LI1 than N2 at $35.6{ }^{\circ} \mathrm{C}$; Additional file 1: Fig. S4CD). No mutants from these sensory classes significantly enhanced locomotory thermal performance at high temperatures, though two trended that way $\left(\operatorname{age}-1\right.$ at $36.7^{\circ} \mathrm{C}$, involved in chemosensation; $g l r-1$ at $37.8^{\circ} \mathrm{C}$, involved in mechanosensation), and 7 of the remaining 20 mutants showed severely compromised TTCs (Additional file 1: Fig. S4C-D). These mutants show that neuro-modulation of thermal performance is not limited to changes in thermosensory genes and cells. Given the limited number of neurons in C. elegans (302 neurons in adult hermaphrodites) [35], many circuits are used for multiple purposes. Even individual sensory neurons may sense temperature as well as other stimuli [36]. The influence of chemo- and mechanosensory genes on TTCs suggests that pleiotropic effects of neuronal disruption might work in the same or parallel circuits to those affected by thermosensory mutants.

Our mutant screen also included six genes involved in dopamine signaling, which is necessary for arousal and activity in C. elegans [37]. In other studies, dop-1 mutants usually have reduced locomotory levels [38], which we also observed (Dunnett's post hoc test $P<0.0001$ at $35.6{ }^{\circ} \mathrm{C}$; Additional file 1: Fig. S4E). Other dopamine pathway mutants showed TTCs similar to the CB4856 Hawaiian wild isolate, which shows reduced locomotory activity at moderately high temperatures $\left(33-36^{\circ} \mathrm{C}\right)$ compared to $\mathrm{N} 2$ (3/6 dopamine mutants lower LI1 than wildtype at $35.6{ }^{\circ} \mathrm{C}$ with Dunnett post hoc test $P<0.004$; Additional file 1: Fig. S4E). Therefore, the changes in locomotory activity with increasing temperature may not involve a major role of dopamine signaling. However, the goa-1 mutant is known for being hyperactive [39], and in our experiment it maintains much faster locomotion than wildtype in the region of the TTC above $35^{\circ} \mathrm{C}$ and has relatively high locomotion even at $37.8^{\circ} \mathrm{C}$ (Dunnett's post hoc test $P<0.0001$ at $37.8{ }^{\circ} \mathrm{C}$; Fig. $3 \mathrm{~b}$ ), where $\mathrm{N} 2$ is nearly motionless. GOA-1 is part of heterotrimeric G-protein alpha subunit $G_{o}$ and, as such, interacts genetically with many other pathways thus making it very pleiotropic, especially with behavior phenotypes. The enhanced activity of goa-1 mutant animals at high temperatures reinforces the idea that the wildtype TTC is not solely a by-product of physiological limits imposed by high temperatures (e.g. muscle function) but reflects in part behavioral decision-making by the animal to modulate locomotory activity.
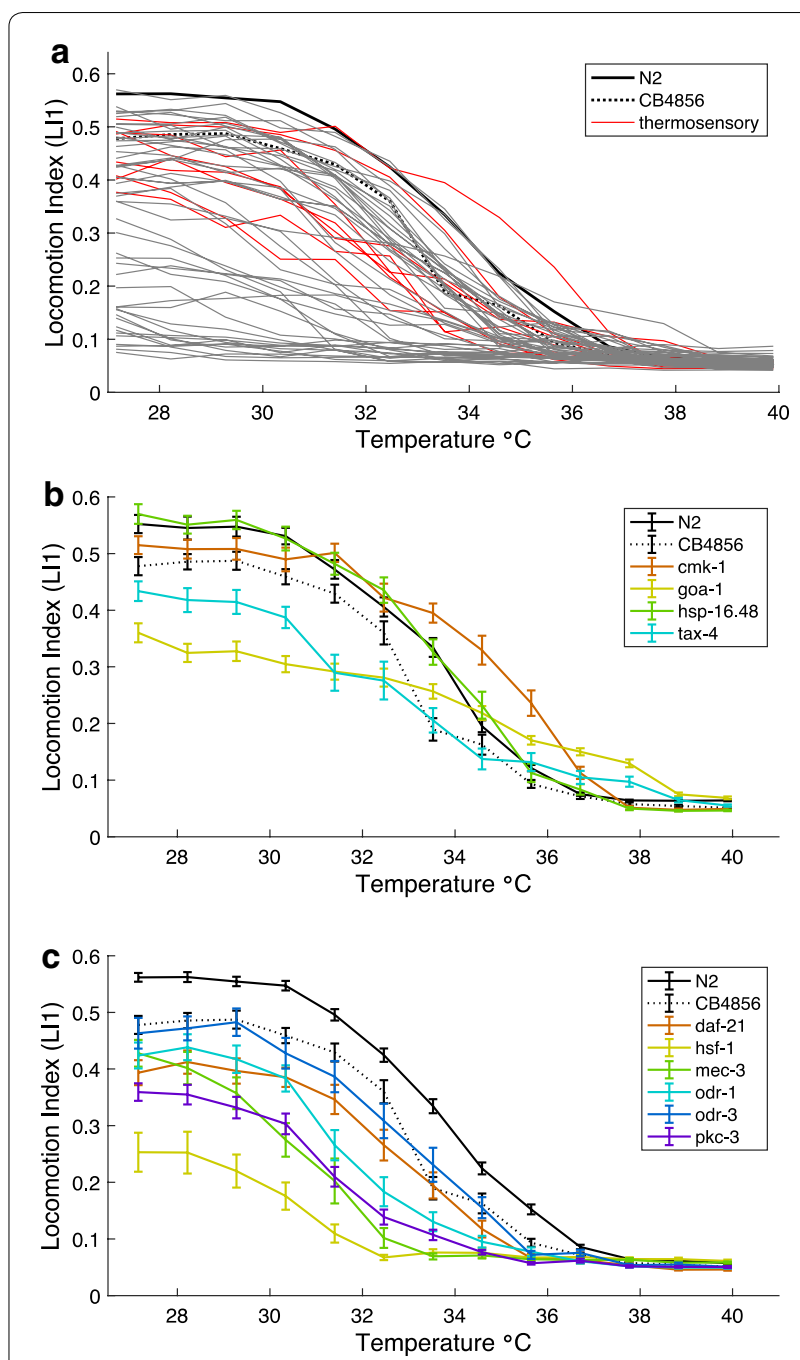

Fig. 3 Effect of mutations on C. elegans TTCS. a Thermal reaction norms of swimming behavior for $58 \mathrm{C}$. elegans genetic mutant strains (gray and red lines) and two wildtype strains (black line $=\mathrm{N} 2$, dashed line $=$ CB4856). All worms reared at $23^{\circ} \mathrm{C} ; 17-36$ individuals included in calculations at each assay step for each strain (Table 1). TTCs for subsets of mutant strains with sensory disruptions from (a) are shown for strains with similar or higher paralysis threshold temperatures than wild-type (b) and strains with lower paralysis temperatures than wild-type (c)

To explore the role of the heat shock response, we tested two heat shock protein mutants (Fig. 3, Additional file 1: Fig. S4F). Only one of two mutants affecting the heat shock response in our screen exhibited an atypical TTC: the $h s f-1$ mutant showed a locomotion deficit even at relatively benign temperatures $\left(27.2^{\circ} \mathrm{C}\right.$ Dunnett's post hoc test $P<0.0001$ ) but became immobile by $\sim 32^{\circ} \mathrm{C}$, an exceptionally low paralysis temperature (Fig. $3 \mathrm{c}, 32.5{ }^{\circ} \mathrm{C}$ Dunnett's post hoc test $P<0.0001)$. Note that the HSP20 chaperone $h s p-16.48$ phenotype was indistinguishable 
from wildtype (Fig. 3b, Dunnett's post hoc test $P=1.0$ at $27.2^{\circ} \mathrm{C}$ and $P=0.55$ at $37.8^{\circ} \mathrm{C}$ ). Even if triggered, however, expression of heat shock pathways may not fully manifest in the timeframe of the assay [40, 41], though pre-conditioning animals to heat stress in advance of assessing TTCs could test for more active roles of heat shock response in behavior, as for survival [23].

\section{Non-neural trpa-1-mediated behavioral plasticity in response to rearing temperature}

The TRPA-1 transient receptor potential ion channel activates at lower environmental temperatures, signaling for changes in gene expression that modulate lifespan in C. elegans [42]. Might trpa-1 also be important in regulating behavioral thermal performance in some way? It is a cold sensitive channel and is involved in thermosensation and mechanosensation in C. elegans [43], but it is also broadly expressed in non-neuronal tissues including the intestine. The TRPA1 family of channels also detect reactive electrophiles and acidification and other nociceptive stimuli [44]. Under our standard locomotion assay conditions (benign $20{ }^{\circ} \mathrm{C}$ rearing temperature), the trpa-1(ok999) knockout mutant strain exhibits modestly reduced locomotory activity compared to the N2 wildtype with equivalent baseline motility. To assess whether this cold-sensing channel might play a deeper role in behavioral thermal performance, we manipulated rearing temperatures of mutant and wildtype trpa-1 alleles and contrasted their TTCs.

We hypothesized that if the downstream targets for trpa-1 signaling affect acclimation behavior, as well as longevity, then trpa-1 knockout animals might show a distinctive sensitivity to rearing conditions. Comparison of TTCs for trpa-1(ok999) in response to different rearing temperatures $\left(15{ }^{\circ} \mathrm{C}, 20{ }^{\circ} \mathrm{C}, 25{ }^{\circ} \mathrm{C}\right)$ revealed that it indeed shows reduced TTC plasticity across rearing conditions (rearing temperature differences for wildtype at $32.5{ }^{\circ} \mathrm{C} \mathrm{F}_{2,147}=36.8, P<0.0001$; for trpa-1 (ok999) at $~ 32$ ${ }^{\circ} \mathrm{C}$ at $32.5^{\circ} \mathrm{C} \mathrm{F}_{2,145}=0.91, P=0.41$; Fig. 4a, b; Additional file 1: Figs. S4, S5). While TTCs for wildtype animals shift toward colder temperatures when reared cold and shift warmer when reared warm, the TTC for the trpa1 mutant strain remains nearly unchanged regardless of rearing temperature (Fig. 4a, b). In other words, knockout of trpa-1 canalizes the TTC, making its shape largely insensitive to rearing conditions. A consequence of the trpa-1 insensitivity to rearing conditions is that trpa1(ok999) animals exhibit enhanced locomotion compared to $\mathrm{N} 2$ at warmer assay temperatures when they are both reared at $15{ }^{\circ} \mathrm{C}$; only rarely do mutants "outperform" the wildtype genotype in our screen of genetic perturbations.

These results are consistent with the hypothesis that trpa-1 mutants do not adjust their behavior normally to rearing temperature. Previous work found that overexpression of TRPA- 1 in neurons and the gut led to longer lifespans at $20{ }^{\circ} \mathrm{C}$, with the gut expression exerting a greater effect [42]. Because we demonstrated that TTCs are at least partly affected by thermosensory neural circuitry, we hypothesized that TRPA-1 expression in neurons might also be important for the mutant phenotype. We therefore next quantified the TTCs for trpa-1 expression rescue lines in a trpa-1 null genetic background, using transgenic constructs with its endogenous promoter or using tissue-specific rescue of expression with neural or gut trpa-1 overexpression lines.

Expressing trpa-1 with its genomic promotor yields a TTC with more plasticity than the gene knockout in response to rearing temperature, especially for cool $15{ }^{\circ} \mathrm{C}$ rearing, indicating at least partial rescue of rearingdependent TTC plasticity (rearing temperature differences for trpa- 1 genomic rescue at $32.5^{\circ} \mathrm{C} \mathrm{F}_{2,140}=17.37$, $P<0.0001$; Fig. 4b; Additional file 1: Figs. S5, S6). Gutspecific expression of trpa-1 also exhibited nearly normal plasticity from rearing temperature, especially for warm $25{ }^{\circ} \mathrm{C}$ rearing (rearing temperature differences for trpa1 gut rescue at $32.5{ }^{\circ} \mathrm{C} \mathrm{F} F_{2,127}=18.41, P<0.0001$; Fig. 4b; Additional file 1: Figs. S5, S6). The imperfect restoration of TTC plasticity might result from over-expression of trpa-1 or the fluorescent markers in the transgenic constructs of the rescue lines that could adversely affect animal health. Interestingly, and counter to our initial predictions, neural-specific trpa-1 expression did not restore any plasticity to the TTCs in response to rearing conditions (rearing temperature differences for trpa1 neural rescue at $32.5{ }^{\circ} \mathrm{C} F_{2,136}=0.90, P=0.41$; Fig. 4b; Additional file 1: Figs. S5, S6), and the TTCs were shifted toward more rapid decline in locomotory activity with ambient temperature changes than for any of the other trpa-1 experimental lines (Fig. 4b; Additional file 1: Fig. S5). Together, these experiments imply that the downstream effects of TRPA-1 thermosensory activity derive from intestinal expression during development, and perhaps expression in other tissues conferred by the endogenous promoter, but not from TRPA-1 thermosensory signaling initiated by neurons.

To further test the idea that rearing-dependent plasticity of TTCs does not depend on neuronal thermosensory cues, we quantified mutant $t t x-1$ (p767) TTCs at $15^{\circ} \mathrm{C}, 20^{\circ} \mathrm{C}$, and $25^{\circ} \mathrm{C}$ rearing conditions. The $t t x-1$ gene encodes a transcription factor necessary for AFD neuron thermosensory fate, and its mutant genotype perceives temperature differently than normal (Fig. 4b). While TTCs for $t$ tx- 1 mutants reared at $20{ }^{\circ} \mathrm{C}$ and $25{ }^{\circ} \mathrm{C}$ were shifted cooler compared to wildtype, the mutant nevertheless exhibited a similar pattern of rearing-dependent plasticity as wildtype N2 (rearing temperature differences 

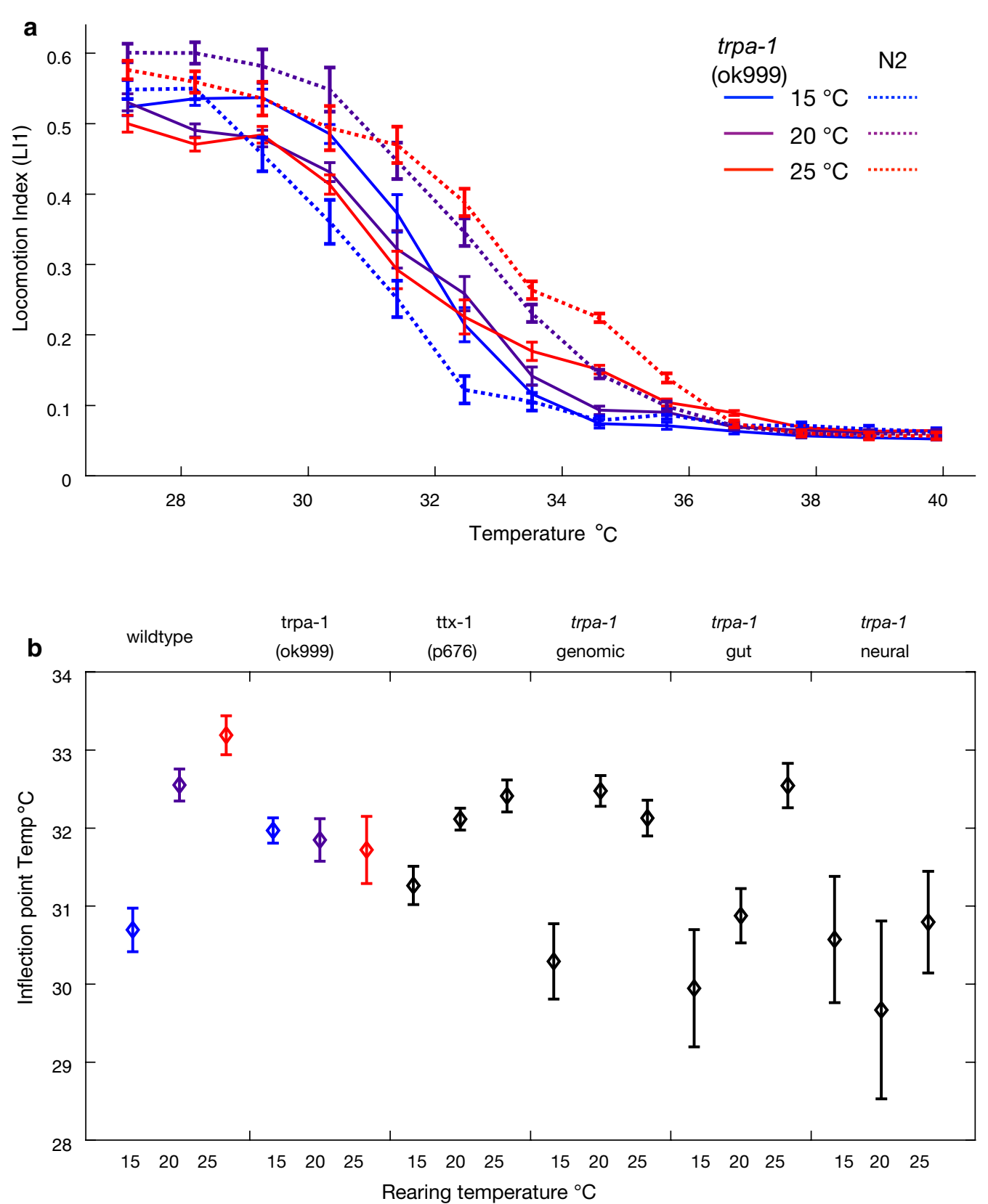

Fig. 4 Comparison of wildtype and trpa-1 mutants over different rearing temperatures. a The sensitivity of the locomotion index TTC to rearing temperature observed for wildtype N2 C. elegans (dashed lines) is reduced for trpa-1 mutant animals (dark lines), which show similar TTCs regardless of rearing conditions. Error bars indicate \pm SEM; 54 animals tested per treatment, $\geq 44$ individuals included in calculations at each assay step. $\mathbf{b}$ Comparison of inflection point estimates for locomotion index TTC curves for wildtype N2, trpa-1 and ttx-1 mutants, and trpa-1 expression-rescue strains (see Table 2 and Additional file 1: Fig. S3). Similar inflection point values for different rearing conditions of a given genotype indicate low TTC plasticity; distinct values for different rearing conditions indicate high TTC plasticity. Genomic and gut expression-rescue constructs for trpa-1 also rescue TTC plasticity, unlike the neural expression-rescue construct. Values for wildtype N2 and trpa-1 (ok999) correspond to TTCs shown in a. Error bars indicate $95 \% \mathrm{Cl}$ from a three-parameter logistic function fit to locomotion index (LI1) values for each strain and rearing temperature; 45-54 animals tested per treatment, $\geq 31$ individuals included in calculations at each assay step

for $t$ tx-1 $(\mathrm{p} 767)$ at $32.5^{\circ} \mathrm{C} \mathrm{F}_{2,139}=9.98, P<0.0001$; Fig. $\left.4 \mathrm{~b}\right)$. This observation reinforces our conclusions from the trpa-1 mutant regarding the notion that neural signaling, and AFD thermosensation in particular, is not involved in controlling plasticity of behavioral TTCs in response to rearing conditions despite the fact that neural control contributes to non-rearing-dependent TTC shape. 
Table 2 trpa-1 construct lines assayed for TTCs

\begin{tabular}{|c|c|c|c|}
\hline Strain $^{a}$ & Construct & Genetics & Explanation \\
\hline RB1052 & - & trpa-1(ok999) & trpa-1 knockout mutant \\
\hline TQ1643 & XuEx601 & XuEx601[Pges-1::trpa-1::SL2::yfp + Punc-122:::DsRed] & Gut promotor overexpression \\
\hline TQ1648 & XuEx606 & XuEx606[Prgef-1::trpa-1::SL2::yfp + Punc-122::DsRed] & Neural promotor over expression \\
\hline TQ2706 & XuEx619 and XuEx735 & $\begin{array}{l}\text { trpa-1 (ok999); lin-15; xuEx735[Pges-1::trpa-1::SL2::-mcherry2 + Punc- } \\
\text { 122delta:::yfp]; xuEx19[Plfe-2L::GCaMP1.3 + Plfe-2L::DsRed2b + lin-15(+)] }\end{array}$ & $\begin{array}{l}\text { trpa-1 rescued with own } \\
\text { genomic DNA promotor }\end{array}$ \\
\hline
\end{tabular}

a Xiao et al. (2013); We tested strain TQ2706 as a trpa-1 rescue using our own upstream genomic promotor. Lines with constructs XuEx601 and XuEx606 were backcrossed separately to strain RB1052 to create tissue-specific trpa-1 rescue lines, confirmed by PCR and fluorescence

\section{Discussion}

We designed and implemented a micro-droplet swimming assay of nematode locomotory behavior to characterize the environmental plasticity and genetic modulation of behavioral reaction norms. These multidimensional data yield phenotypic functional responses that take the form of thermal tolerance curves (TTC) to capture distinct thermal performance profiles among natural isolates, mutant alleles, and rearing conditions. By screening TTCs for 58 C. elegans mutant strains with known or suspected roles in sensory perception and locomotion, we found potential neural controls that contribute to TTC shape. Mutations to thermosensory pathways are capable of shifting the decision-making process to cause animals to continue swimming at higher temperatures or to cease moving at lower temperatures than wild-type worms. These experiments provide evidence for a neural "decision" to stop locomotion toward the upper portions of thermal tolerance curves, in addition to physiological limits of high temperature stress on locomotory activity. In addition to neural components, we also show that trpa-1 expression in non-neural tissue affects the plasticity of the TTC.

Previous work on thermotaxis $[15,45]$ and thermal avoidance [46] has shown that C. elegans thermal behavior can be modified by experience. In a similar fashion, through behavior, the animal can "choose" to not perform at its peak, and the less-than-peak performance might be optimal for the organism. In this way, behavioral TTCs in our experiments differ in part from "classic" thermal performance curves of maximum sprint speed or fecundity that test absolute physiological thermal limits of the organism [12, 21]. Even with neural control over temperature-dependent behavior, however, disrupted homeostasis must also contribute to TTC profiles at extreme temperatures. Nevertheless, our observations from sensory gene mutants that swim actively at temperatures even more extreme than wildtype argue that hard cell physiological limitation is not the sole determinant of locomotory declines in C. elegans.
Reduced locomotory activity of C. elegans from elevated temperature depends on continuous exposure to temperatures above a threshold near $30^{\circ} \mathrm{C}$ and exposure to just $80 \mathrm{~s}$ of extreme temperatures $>39{ }^{\circ} \mathrm{C}$ can alter locomotory behavior for at least an hour. Moreover, plasticity in animal TTC profiles derives from differences in age and, most importantly, the temperature of rearing conditions. We demonstrated that this TTC plasticity due to rearing conditions also has a genetic basis, such that knockout of the trpa-1 ion channel ablates the normal sensitivity that adult $C$. elegans have to the rearing temperature conditions they experienced as larvae, resulting in a 'canalized' behavioral response.

Strikingly, it is a non-neural component of thermal tolerance regulation that mediates the trpa-1-dependent sensitivity of TTC profiles to rearing conditions. Previous work demonstrated that cold sensing of the C. elegans intestine regulates lifespan [42], and here we show that intestinal expression of trpa-1 affects sensorimotor responses such as thermal tolerance. Thus, normal thermal response behaviors depend crucially on integration and feedback between distinct tissue types, including neurons that contribute to TTC shape and intestinal cells that contribute to how the animal assimilates 'memory' of rearing conditions. Other non-neural tissues also are reported to affect thermal behavior in C. elegans, with even sperm cells shown to influence thermal performance of the organism [47]. In addition, hormone signals from many other tissues affect general worm locomotory behavior, including insulin or steroidal signaling [48, 49]. It is clear that we are only beginning to understand how the whole body of $C$. elegans is integrated to regulate its sensory behavior.

Viewed through the lens of adaptive phenotypic plasticity [50], the "decision" to stop moving at high temperatures likely reflects some kind of preventative or protective response to improve fitness. Indeed, studies showing that mutants with less quiescence after exposure to high temperature have lower survival rates suggests that cessation of locomotion may be important for recovery from or coping with thermal stress [22]. Given that normal functioning of trpa-1 confers TTC plasticity to 
rearing conditions, the extent to which the sensitivity of TTCs to rearing conditions are adaptive would imply that trpa-1 represents an "adaptive plasticity" gene. Future work that connects behavior to fitness of the trpa-1 mutants at different temperatures, including low temperatures, would also help determine whether the plasticity that gets ablated by knockout of trpa-1 is related to any adaptive role for trpa-1 signaling.

\section{Conclusion}

Understanding the environmental plasticity and genetic evolvability of the thermal ecology for ectothermic organisms is fundamental, given how temperature exerts profound effects on all levels of biological function, with special urgency due to the effects of recent climate change. Here we developed an assay to quantify the thermal tolerance of C. elegans across an ecologically relevant temperature range. Through a canvasing of candidate genes, we show that the thermal performance of C. elegans is sensitive to genetic perturbations of both behavioral decision-making and physiological limits.

\section{Materials and methods}

\section{Worm strains and preparation}

We reared 58 isogenic strains each carrying a homozygous mutation plus two "wildtype" strains of C. elegans using standard protocols [51] (Table 1), obtained from the Caenorhabditis Genetics Centre (University of Minnesota, Minneapolis, MN, USA) which is funded by $\mathrm{NIH}$ Office of Research Infrastructure Programs (P40 OD010440) with some mutants created by the C. elegans knockout consortium [52]. We selected strains based on published literature that indicated roles in thermotaxis, thermosensation, mechanosensation, chemosensation, locomotion, and neural function. All mutant strains derive from the $\mathrm{N} 2$ genetic background, so $\mathrm{N} 2$ represents the primary control for comparison. We assayed two common wild-derived C. elegans strains as controls: the archetypal N2 strain (Bristol, England) and the genetically distinct CB4856 (Hawaii, USA), which have been used in QTL mapping of other temperature-dependent traits and behaviors [53-57]. These strains provide a useful reference for the potential natural range in phenotype without disruptive gene mutations. Behavior assays used only well-fed adult worms, except in experiments with dauer, L3 and L4 stage larvae. For rearing temperature manipulations, we raised all worms from eggs at the desired temperature from parents raised at the same temperature.

\section{Micro-droplet assay of thermal behavioral responses}

We constructed an experimental apparatus to quantify nematode locomotory behavior (Additional file 1: Fig. S2) across a wide range of temperature. The device consists of a high-resolution video camera (Allied vision technology, model GX3300 with Nikon AF-Nikkor 80 mm lens) mounted above a temperature-controlled aluminum stage $(165 \mathrm{~mm} \times 58 \mathrm{~mm} \times 4.5 \mathrm{~mm})$. The imaging stage is obliquely illuminated by two LED strips. Two thermoelectric control devices (TEC), cooled by liquid CPU coolers (Swiftech, MCW30; Thermo Scientific, NESLAB Digital One RTE7), control the temperature of the stage. A custom-built closed-loop input/output controller, driven by a program written in LabVIEW (National Instruments, Texas) controls the TECs, and a thermocouple $\left( \pm 0.1{ }^{\circ} \mathrm{C}\right)$ measures the stage temperature near the stage center.

To assess nematode behavior on the instrument stage, we placed individual worms within $\sim 2 \mu \mathrm{L}$ NGM (nematode growth medium) liquid droplets on a Teflon (PTFE) printed microscope slide that hydrophobically constrains the droplets in a 2D array (Tekdon slide ID: 24-20). Worms were distributed in the middle $3 \times 6$ wells of a slide patterned with a $3 \times 8$ array of $4 \mathrm{~mm}$ diameter wells. The slide was sealed with a coverslip that contacted the NGM droplets, using two layers of double-sided sticky tape and M10 Apiezon vacuum grease along its edges.

\section{Experimental protocol for behavioral quantification}

Using a worm pick, worms from stock plates were placed into NGM buffer at room temperature, swirled to wash off bacteria and poorly swimming worms were removed. We pipetted $1.8 \mu \mathrm{L}$ of NGM buffer containing a single worm into each of 18 wells per slide $(\sim 7 \mathrm{~min}$ total prep time), with each half of the slide holding a randomized pair of strains for experiments comparing different genotypes.

Our standard experimental assay comprised of sixteen, $\sim 1{ }^{\circ} \mathrm{C}$ temperature steps, of $80 \mathrm{~s}$ duration, where we let the system to come to equilibrium for $60 \mathrm{~s}$ and then captured $20 \mathrm{~s}$ of behavioral video at $15 \mathrm{fps}(3296 \times 1600$ pixels). Image capture and primary analysis used custom software written with LabVIEW. For each video frame, the analysis program identifies the worm in each droplet using particle detection and measures its area and center-of-mass position. Worm size and swimming speed were used as criteria for removing data affected by poor worm tracking or abnormally moving worms. Cutoffs include a maximum allowable worm size (mean area 375 pixels, maximum area 803 pixels), a maximum percentage change in worm size (31\% between adjacent frames threshold value) and a maximum change in worm position between frames (15 'pixel' width, threshold value), determined from a pilot study of 198 animals from two C. briggsae strains (AF16 and HK104; similar size and 
behavior as C. elegans). Each temperature step recording required at least 100 of the possible 300 frames of data in order to be included in downstream analysis.

We define a "Locomotion Index" (LI) to quantify movement activity by comparing worm images between video frames. The LI is calculated as the ratio of the number of non-overlapping worm pixels between two frames over the total number of worm pixels for the two frames. When a worm moves slowly between frames, nearly all pixels will overlap and the LI will be close to 0 . Fast worm movement results in less pixel overlap and the LI will approach a maximum value of 1 . In practice, stochastic pixel noise produces a non-zero lower bound of LI values $(\sim 0.05)$ and the partial overlap of worm position between frames yields an upper bound ( $\sim 0.60)$. We did not normalize LI within strains in order to capture differences in the baseline. We indicate the sampling rate of the LI as LI\#, where \# indicates the index of the second frame used to calculate the LI (e.g. LI1 indicates successive frames, while LI7 indicates every 7th frame). Both LI1 and LI7 measurements gave qualitatively similar results, but we chose one over the other depending on how slow the animals were moving. We determined whether animals were moving or not based on a threshold of the mean locomotion index calculated seven frames apart (LI7, $0.47 \mathrm{~s}$ resolution). Only $2.5 \%$ of worms exceeded the threshold value at extreme temperatures ( LI7 $>0.14)$, when all worms are paralyzed, using the same dataset used to calculate quality control cut-offs.

We determined statistical significance of differences between strain performance with one-way ANOVA and post hoc tests at particular temperatures in the thermal response. ANOVA post hoc tests used either Dunnett's test with N2 as control [58] or Tukey-Kramer Honestly Significant Differences (HSD). In comparisons of plasticity for trpa-1 experiments, we also tested for overlap of 95\% CI for parameters from a three parameter logistic function fit to LI using non-linear model fitting in JMP 10.0.0: $I(T)=\frac{\alpha}{1+e^{(-\beta \cdot(T-\tau))}}$, where $T$ is droplet temperature and $\tau$ is the inflection point temperature in the TTC.

\section{Additional file}

Additional file 1. Supplementary figures.

\section{Abbreviations}

ANOVA: analysis of variance; CGC: caenorhabditis genetics center; CPU: computer processing unit; NGM: nematode growth medium; PTFE: polytetrafluoroethylene; TEC: thermal electric cooler; TTC: thermal tolerance curve.

\section{Acknowledgements}

We thank Jiwon Shin for preliminary work on the droplet assay. Some strains were provided by the CGC, which is funded by NIH Office of Research Infrastructure Programs (P40 0D010440).

\section{Authors' contributions}

GWS, ADC, and WSR wrote the manuscript and designed the experiments. GWS and DM performed the experiments. GWS performed the data analysis. GWS and ADC performed the statistical testing. All authors read and approved the final manuscript.

\section{Funding}

ADC was supported by funds from the Natural Sciences and Engineering Council of Canada and a Canada Research Chair. WSR was supported by funds from Natural Sciences and Engineering Council of Canada.

\section{Availability of data and materials}

All data analyzed for this paper are available from the corresponding authors on reasonable request.

\section{Ethics approval and consent to participate}

Not applicable.

\section{Consent for publication}

Not applicable.

\section{Competing interests}

The authors declare that they have no competing interests.

\section{Author details}

${ }^{1}$ Department of Ecology and Evolutionary Biology, University of Toronto, Toronto, Canada. ${ }^{2}$ Department of Physics, University of Toronto, Toronto,

Canada. ${ }^{3}$ Donnelly Centre, University of Toronto, Toronto, ON M5S3E1, Canada.

Received: 8 April 2019 Accepted: 3 June 2019

Published online: 10 June 2019

\section{References}

1. Lorenz KZ. The evolution of behavior. Sci Am. 1958;199(6):67-82.

2. Fitzpatrick MJ, Ben-Shahar Y, Smid HM, Vet LE, Robinson GE, Sokolowski MB. Candidate genes for behavioural ecology. Trends Ecol Evol. 2005;20(2):96-104.

3. Boake CRB, Arnold SJ, Breden F, Meffert LM, Ritchie MG, Taylor BJ, et al. Genetic tools for studying adaptation and the evolution of behavior. Am Nat. 2002;160:5143-59.

4. Anholt RR, Mackay TF. Quantitative genetic analyses of complex behaviours in Drosophila. Nat Rev Genet. 2004;5(11):838-49.

5. Fuller JL. Behavior genetics. Annu Rev Psychol. 1960;1 1 (1):41-70.

6. Gavrilets S, Scheiner SM. The genetics of phenotypic plasticity. Evolution of reaction norm shape. J Evol Biol. 1993;6(1):31-48.

7. Dingemanse NJ, Kazem AJN, Réale D, Wright J. Behavioural reaction norms: animal personality meets individual plasticity. Trends Ecol Evol. 2010;25(2):81-9.

8. Huey RB, Kingsolver JG. Evolution of thermal sensitivity of ectotherm performance. Trends Ecol Evol. 1989;4(5):131-5.

9. Huey RB, Stevenson RD. Integrating thermal physiology and ecology of ectotherms: a discussion of approaches. Am Zool. 1979;19(1):357-66.

10. Angilletta MJ. Thermal adaptation. New York: Oxford University Press; 2009.

11. Garrity PA. Role of adaptation in C. elegans thermotaxis. Focus on "Shortterm adaptation and temporal processing in the cryophilic response of Caenorabditis elegans". J Neurophysiol. 2007;97(3):1874-6.

12. Huey RB, Kingsolver JG. Evolution of resistance to high-temperature in ectotherms. Am Nat. 1993;142:S21-46.

13. Sokolowski MB. Drosophila: genetics meets behaviour. Nat Rev Genet. 2001:2:879.

14. Hedgecock EM, Russell RL. Normal and mutant thermotaxis in the nematode Caenorhabditis elegans. Proc Nat Acad Sci USA. 1975;72(10):4061-5.

15. Wittenburg N, Baumeister R. Thermal avoidance in Caenorhabditis elegans: an approach to the study of nociception. Proc Natl Acad Sci USA. 1999;96(18):10477-82.

16. Schild LC, Glauser DA. Dynamic switching between escape and avoidance regimes reduces Caenorhabditis elegans exposure to noxious heat. Nat Commun. 2013;4:2198. 
17. Aoki I, Mori I. Molecular biology of thermosensory transduction in C. elegans. Curr Opin Neurobiol. 2015;34:117-24.

18. Goodman MB, Klein M, Lasse S, Luo L, Mori I, Samuel ADT, et al. Thermotaxis navigation behavior. Wormbook; 2014. https://doi.org/10.1895/ wormbook.1.168.1. www.wormbook.org.

19. Buckingham SD, Sattelle DB. Strategies for automated analysis of $C$. elegans locomotion. Invertebr Neurosci. 2008:8(3):121.

20. Restif C, Metaxas D. A comparison of tracking methods for swimming C. elegans. SPIE Med Imaging. 2010;7623:1-8.

21. Angilletta MJ, Niewiarowski PH, Navas CA. The evolution of thermal physiology in ectotherms. J Therm Biol. 2002;27(4):249-68.

22. Hill Andrew J, Mansfield R, Lopez Jessie MNG, Raizen David M, Van Buskirk C. Cellular stress induces a protective sleep-like state in C. elegans. Curr Biol. 2014;24(20):2399-405.

23. Kourtis N, Nikoletopoulou V, Tavernarakis N. Small heat-shock proteins protect from heat-stroke-associated neurodegeneration. Nature. 2012;490:213.

24. Gutteling EW, Riksen JA, Bakker J, Kammenga JE. Mapping phenotypic plasticity and genotype-environment interactions affecting life-history traits in Caenorhabditis elegans. Heredity. 2007;98(1):28-37.

25. Harvey SC, Viney ME. Thermal variation reveals natural variation between isolates of Caenorhabditis elegans. J Exp Zool. 2007;308B:409-16.

26. Ryu WS, Samuel AD. Thermotaxis in Caenorhabditis elegans analyzed by measuring responses to defined thermal stimuli. J Neurosci. 2002;22(13):5727-33.

27. Garrity PA, Goodman MB, Samuel AD, Sengupta P. Running hot and cold: behavioral strategies, neural circuits, and the molecular machinery for thermotaxis in C. elegans and Drosophila. Genes Dev. 2010;24(21):2365-82.

28. Grewal PS. Influence of bacteria and temperature on the reproduction of Caenorhabditis elegans (Nematoda, Rhabditidae) infesting mushrooms (Agaricus bisporus). Nematologica. 1991;37(1):72-82.

29. Petrella LN. Natural variants of C. elegans demonstrate defects in both sperm function and oogenesis at elevated temperatures. PLOS ONE. 2014:9(11):e112377.

30. Cassada RC, Russell RL. The dauerlarva, a post-embryonic developmental variant of the nematode Caenorhabditis elegans. Dev Biol. 1975;46(2):326-42.

31. Golden JW, Riddle DL. The Caenorhabditis elegans dauer larva - developmental effects of pheromone, food, and temperature. Dev Biol. 1984;102(2):368-78.

32. de Bono $\mathrm{M}$, Bargmann $\mathrm{Cl}$. Natural variation in a neuropeptide $\mathrm{Y}$ receptor homolog modifies social behavior and food response in C. elegans. Cell. 1998;94(5):679-89.

33. Komatsu H, Mori I, Rhee J-S, Akaike N, Ohshima Y. Mutations in a cyclic nucleotide-gated channel lead to abnormal thermosensation and chemosensation in C. elegans. Neuron. 1996;17(4):707-18.

34. Satterlee JS, Ryu WS, Sengupta P. The CMK-1 CaMKI and the TAX-4 cyclic nucleotide-gated channel regulate thermosensory neuron gene expression and function in C. elegans. Curr Biol. 2004;14(1):62-8.

35. White JG, Southgate E, Thomson JN, Brenner S. The structure of the nervous system of the nematode Caenorhabditis elegans. Philos Trans R Soc Lond Ser B Biol Sci. 1986;314(1165):1-340.

36. Biron D, Wasserman S, Thomas JH, Samuel ADT, Sengupta P. An olfactory neuron responds stochastically to temperature and modulates Caenorhabditis elegans thermotactic behavior. Proc Natl Acad Sci USA 2008;105:11002-7.

37. Singh K, Ju JY, Walsh MB, Dilorio MA, Hart AC. Deep conservation of genes required for both Drosophila melanogaster and Caenorhabditis elegans sleep includes a role for dopaminergic signaling. Sleep. 2014;37(9):1439-51.

38. Keating CD, Kriek N, Daniels M, Ashcroft NR, Hopper NA, Siney EJ, et al. Whole-genome analysis of $60 \mathrm{G}$ protein-coupled receptors in Caenorhabditis elegans by gene knockout with RNAi. Curr Biol. 2003;13(19):1715-20.
39. Mendel JE, Korswagen HC, Liu KS, Hajdu-Cronin YM, Simon MI, Plasterk $\mathrm{RH}$, et al. Participation of the protein Go in multiple aspects of behavior in C. elegans. Science. 1995;267(5204):1652.

40. Snutch TP, Baillie DL. Alterations in the pattern of gene expression following heat shock in the nematode Caenorhabditis elegans. Can J Biochem Cell Biol. 1983;61(6):480-7.

41. Dawe AS, Smith B, Thomas DWP, Greedy S, Vasic N, Gregory A, et al. A small temperature rise may contribute towards the apparent induction by microwaves of heat-shock gene expression in the nematode Caenorhabditis elegans. Bioelectromagnetics. 2006;27(2):88-97.

42. Xiao R, Zhang B, Dong Y, Gong J, Xu T, Liu J, et al. A genetic program promotes $C$. elegans longevity at cold temperatures via a thermosensitive TRP channel. Cell. 2013;152(4):806-17.

43. Kindt KS, Viswanath V, Macpherson L, Quast K, Hu H, Patapoutian A, et al. Caenorhabditis elegans TRPA-1 functions in mechanosensation. Nat Neurosci. 2007;10:568.

44. Garrity PA. Weakly acidic, but strongly irritating: TRPA1 and the activation of nociceptors by cytoplasmic acidification. J Gen Physiol. 2011;137(6):489.

45. Goodman MB, Sengupta P. The extraordinary AFD thermosensor of $C$. elegans. Pflugers Arch. 2018;470(5):839-49.

46. Schild LC, Zbinden L, Bell HW, Yu YXV, Sengupta P, Goodman MB, et al. The balance between cytoplasmic and nuclear CaM Kinase-1 signaling controls the operating range of noxious heat avoidance. Neuron. 2014;84(5):983-96

47. Sonoda S, Ohta A, Maruo A, Ujisawa T, Kuhara A. Sperm affects head sensory neuron in temperature tolerance of Caenorhabditis elegans. Cell Rep. 2016;16(1):56-65.

48. Yamawaki TM, Berman JR, Suchanek-Kavipurapu M, McCormick M, et al. The somatic reproductive tissues of $C$. elegans promote longevity through steroid hormone signaling. PLoS Biol. 2010;8(8):e1000468.

49. Murphy CT, Hu PJ. Insulin/insulin-like growth factor signaling in C. elegans. Wormbook; 2013: https://doi.org/10.1895/wormbook.1.164.1.

50. Gotthard K, Nylin S. Adaptive plasticity and plasticity as an adaptation: a selective review of plasticity in animal morphology and life history. Oikos. 1995;74(1):3-17.

51. Stiernagle TL. Maintenance of C. elegans. In: Hope IA, editor. C. elegans: A Practical Approach. New York: Oxford University Press; 1999.

52. C. elegans Deletion Consortium. Large-scale screening for targeted knockouts in the Caenorhabditis elegans genome. G3: Genes|Genomes|Genetics. 2012;2(11):1415.

53. Doroszuk A, Snoek LB, Fradin E, Riksen J, Kammenga J. A genome-wide library of CB4856/N2 introgression lines of Caenorhabditis elegans. Nucleic Acids Res. 2009;37(16):e110.

54. Kammenga JE, Phillips PC, De Bono M, Doroszuk A. Beyond induced mutants: using worms to study natural variation in genetic pathways. Trends Genet. 2008;24(4):178-85.

55. Choi S, Chatzigeorgiou M, Taylor Kelsey P, Schafer William R, Kaplan Joshua M. Analysis of NPR-1 reveals a circuit mechanism for behavioral quiescence in C. elegans. Neuron. 2013;78(5):869-80.

56. McGrath PT, Rockman MV, Zimmer M, Jang H, Macosko EZ, Kruglyak L, et al. Quantitative mapping of a digenic behavioral trait implicates globin variation in C. elegans sensory behaviors. Neuron. 2009;61(5):692-9.

57. Reddy KC, Andersen EC, Kruglyak L, Kim DH. A polymorphism in npr-1 is a behavioral determinant of pathogen susceptibility in C. elegans. Science. 2009;323(5912):382

58. Dunnett CW. A multiple comparison procedure for comparing several treatments with a control. J Am Stat Assoc. 1955;50(272):1096-121.

\section{Publisher's Note}

Springer Nature remains neutral with regard to jurisdictional claims in published maps and institutional affiliations. 